\title{
EFSUMB Newsletter meets Latvia
}

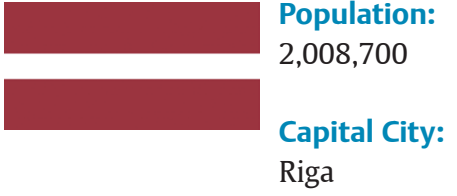

EFSUMB members: 180

The interview with the representative from the Latvian Society of Ultrasound (LUSA), President Maija Radzina took place in May 2014 with Newsletter Editor Adrian Saftoiu.

\section{History of the Latvian Society of Ultrasound \\ $\nabla$}

The Latvian Society of Ultrasound (LUSA) was established on June 5th, 2003. Ilze Sedleniece was elected President of the association in a general meeting of founders, and served till 2011 when the current President, Maija Radzina took over. As of now, the LUSA unites almost 200 members. On June 5th, 2004 during the Congress of European Federation of Societies for Ultrasound in Medicine and Biology EUROSON 2004 in Zagreb the Latvian Society of Ultrasound was approved as a member of European Federation of Societies for Ultrasound in Medicine and Biology (EFSUMB). Since 2004, delegations of LUSA members of around 40-50 people are complementing their knowledge each year by attending the Congress of Euro- pean Ultrasound EUROSON. Also this year EUROSON 2014 will be attended by 40 members of LUSA and we find it as the best networking and collaboration platform that bonds different professionals in the field and has become as tradition.

\section{Ultrasound training in Latvia $\nabla$}

Since 2003 LUSA is hosting bi-yearly conferences (last week of November and May) for its members on different subjects or organising joint meetings with other professional societies - vascular surgery, urology, endocrinology. On a regular basis different basic and advanced ultrasound courses are organised for different specialty doctors who are beginners or advanced users of the method. There are several on-going national courses now: General US, Vascular US, Nerve US, Musculoskeletal US, Breast US.

The ultrasound method certification process is supervised by national Radiology association and national Medical association in Latvia. All ultrasound examinations are performed only by doctors and radiologists. The training curriculum includes all ultrasound skills.

\section{National Congresses and Courses $\nabla$}

There is also significant international activity in collaboration with different in-

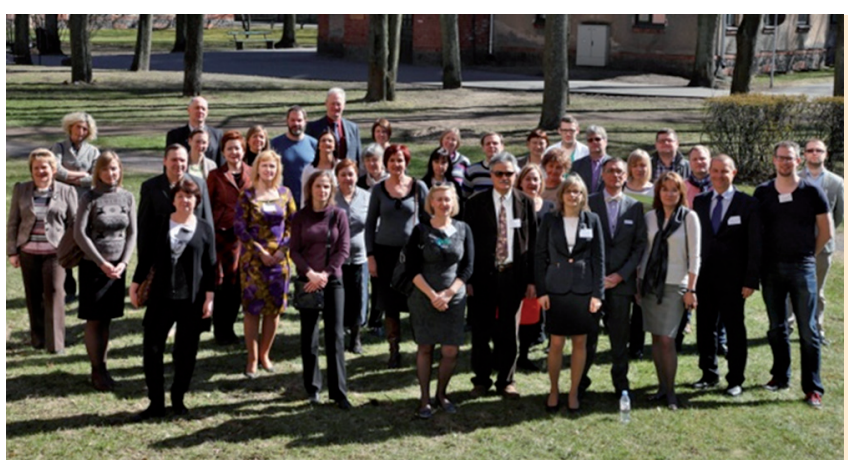

2014 Elastography and Interventional Ultrasound Course organisers and participants, Riga, Latvia

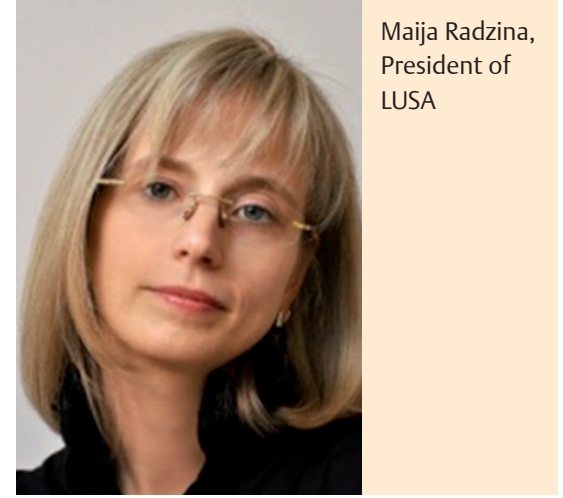

ternational organisations and peers from other countries in organising different courses:

- 2004 IBUS (International Breast Ultrasound School) Advanced Breast Imaging Seminar

- 2008 Course of lectures on brachiocephalic, transcranial, peripheral and abdominal Doppler ultrasound, by prof. Svetlana Leljuka (Moscow, Russia)

- 2009 SIS (Senologic International Society) Breast Imaging Course by prof. Ivan Drinkovitsch (Croatia), prof. Tibor Tot (Sweden) and Dominique Ami (France)

- 2010 Several 3 day courses in regions of Latvia for doctors of all specialties "Interpretation of Ultrasound Imaging for Non-Radiologists" by Maija Radzina and Ilze Sedleniece. More than 300 professionals were trained with the support of ESF project "Further Education of Personnel in Health Care and Health Promotion Institutions for the Purpose of Sustainable Growth of the Industry" 
- 2011 A 3 day course "Ultrasonography in Pediatrics" by Hungarian specialists Prof. Zoltan Harkanyi and George Harmat with an active participation of pediatricians and pediatric US specialists Dace Kreicsteine and Arturs Plume. More than 100 doctors were trained with support of ESF project "Further Education of Personnel in Health Care and Health Promotion Institutions for the Purpose of Sustainable Growth of the Industry"

- 2012 Contrast enhanced ultrasound course with 41 participants. Course was lead by Prof. V Valek and his colleagues M Mechl , A Šprlakova, $\mathrm{H}$ Petrašova and S Bohata. (Brno, Czech Republic)

- 2012 2D, 3D, 4D Pelvic Floor Ultrasound Course. Course by Prof. Andrzej Pawel Wieczorek, 52 participants

- The most recent Elastography and Interventional ultrasound course was held on April 4-5, 2014. This EFSUMB endorsed course included lecturers from France, Greece, Russia, Denmark, Poland and Latvia, with 66 participants.

\section{What do members expect from EFSUMB \\ $\nabla$}

Our collaboration with EFSUMB is growing year by year and we actively participate in EUROSON Schools and EUROSON congresses with contribution of our lecturers and workshop consultants in collaboration with Hellenic Society for Ultrasound in Medicine and Biology and Israel Society for Diagnostic Ultrasound in Medicine. Our experienced colleague Peteris Prieditis will give the EUROSON lecture on thyroid biopsies and Maija Radzina will participate in hands-on sessions as well as co-chairing congress sessions at EUROSON 2014. We also are looking forward to collaborating with other associations as well.

Our positive experiences prove that physical and geographical distance or various historical backgrounds are no barriers if we have the same vision of educational and professional development and work together as a team.

\section{Website}

$\nabla$

Considering the size of Latvia it is impressive that Latvians are listed 28th in the worldwide statistics of EFSUMB website users and clearly English is no obstacle to using this site. Maija Radzina is also a Russian speaker and will be translating the Case of the Month into both Russian and Latvian for EFSUMB. Latvia has its own website www.ultrasonografija.lv

\section{Riga Capital of Culture 2014}

Riga is one of the largest cities on shores of the Baltic Sea with a rich cultural and historic heritage and Maija encourages us all to visit and enjoy the wonderful capital of Lativa. 